\title{
Padrão espacial da castanheira em florestas no sul do Amapá, Amazônia Oriental
}

\section{Spatial pattern of brazilnut tree in forests in the south Amapá state, Eastern Amazon}

\author{
Vitória Nádia Andrade da Fonseca ${ }^{1}$, Rafael Lucas Figueiredo de Souza ${ }^{2 *}$, Dayane Nathália \\ Barbosa Pastana ${ }^{3}$, Marcelino Carneiro Guedes ${ }^{4}$, Anderson Pedro Bernardina Batista ${ }^{5}$
}

'Universidade do Estado do Amapá, Macapá, Amapá, Brasil-fonseca.vna@gmail.com

${ }^{2}$ Universidade Federal de Lavras, pós-graduação em Ciências Florestais, Lavras, Minas Gerais, Brasil - rafaelflorestal55@gmail.com *Autor para correspondência

${ }^{3}$ Universidade Federal de Lavras, pós graduação em Ciências Florestais, Lavras, Minas Gerais, Brasil - dayanepastanaa@gmail.com

${ }^{4}$ Empresa Brasileira de Pesquisa Agropecuária EMBRAPA, Doutor em Recursos Florestais, Macapá, Amapá, Brasil marcelino.guedes@embrapa.br

IInstituto Federal do Amazonas - IFAM, Doutor em Ciências Florestais, São Gabriel da Cachoeira, Manaus, Brasil anderson_pedro22@yahoo.com.br

\section{Palavras-chave \\ Manejo florestal Estatística espacial pontual Extrativismo}

\section{Keywords}

Forest management Spatial statistics Extractivism

\begin{abstract}
A castanha-da-amazônia (Bertholletia excelsa Bonpl.) é uma espécie de grande concentração na Amazônia brasileira e possui grande valor cultural, social e econômico por se tratar de uma espécie muito utilizada por comunidades extrativistas da região. Sabe-se que indivíduos de uma determinada espécie podem apresentar diferentes padrões de distribuição espacial dentro de uma população vegetal, esses padrões estão correlacionados com fatores bióticos e abióticos e o seu entendimento é necessário para o auxílio de planejamento e manejo florestal. $\mathrm{O}$ objetivo deste trabalho foi identificar um padrão espacial das árvores de castanha-da-amazônia na floresta nativa no sul do Amapá. Foram implantadas três parcelas permanentes de $300 \mathrm{~m}$ x 300 metros na floresta madura de terra firme na Reserva Extrativista do Rio Cajari (RESEX Cajari), para verificar o padrão de distribuição espacial da espécie serão testadas as funções: $\mathrm{F} \mathrm{e} \mathrm{K}$. No levantamento foram amostrados 169 indivíduos de castanha-da-amazônia nas três parcelas permanentes e a análise da completa aleatoriedade espacial foi confirmada em ambas parcelas nas duas funções testadas, e esse padrão pode ser explicado pela ação dos dispersores naturais. Não houve diferença no padrão de distribuição nas áreas estudadas, porque o extrativismo da castanha na região selecionou um grande número de indivíduos adultos nos castanhais por conta do diâmetro mínimo de produção $(\geq 50 \mathrm{~cm})$. Entretanto, por conta da dispersão zoocórica, novos indivíduos de castanheira podem estar se desenvolvendo em regiões mais abertas, por conta da maior disponibilidade de luz e preferência dos dispersores.
\end{abstract}

Brazil nut are a species of great concentration in the Brazilian Amazon and have great cultural, social and economic value because it is a species widely used by extractive communities in the region. It is known that individuals of a certain species can present different patterns of spatial distribution within a plant population, these patterns are correlated with biotic and abiotic factors and their understanding is necessary for the aid of planning and forest management. The objective of this work is to identify a spatial pattern of Amazonian nut trees in the native forest in southern Amapá. Three permanent plots of $300 \mathrm{mx} 300 \mathrm{~m}$ were implanted in the mature landland forest in the Cajari River Extractive Reserve (Cajari RESEX), to verify the spatial distribution pattern of the species, the functions $F$ and $K$ were tested. (Bertholletia excelsa Bonpl.) in the three permanent plots in mature terra firme forest and the analysis of the complete spatial randomness was confirmed in both plots in the two functions tested, and this pattern can be explained by the action of the natural dispersers. There was no difference in the distribution pattern in the studied areas, because the Brazil nut extractivism in the region selected a large number of adult individuals in the brazil nut because of the minimum diameter of production $(\geq 50 \mathrm{~cm})$. However, because of the zoocoric dispersion, new individuals of Brazil nut may be developing in more open regions, due to the greater availability of light and preference of the dispersers.

\section{INTRODUÇÃO}

A castanha-da-amazônia (Bertholletia excelsa Bonpl.), conhecida popularmente como castanha do Pará ou castanha do Brasil é encontrada em vários países na América do Sul, mas se concentra na Amazônia legal (NEVES et al., 2015). Possui grande valor cultural, social e econômico por se tratar de uma espécie muito utilizada por comunidades tradicionais 
na reserva agroextrativista do Rio Cajari como principal fonte de renda (TONINI et al., 2018).

O fruto da $B$. excelsa é um produto florestal nãomadeireiro de ampla aceitação no mercado nacional e internacional. A exportação há mais de um século, constituindo-se na colheita de sementes feita exclusivamente em florestas tropicais primárias (MARTINS et al., 2018). No entanto, sem a preservação deste importante produto extrativista amazônico, poderá levar esta atividade a um declínio (SANTANA et al., 2018).

Os estudos sobre a distribuição espacial são ferramentas fundamentais para compreender a dinâmica ecológica e tornar sustentável o uso dos recursos naturais de uma espécie, uma vez que os padrões de distribuição espacial podem influenciar na ecologia da comunidade (LIMA et al., 2018), O comportamento do padrão espacial em diferentes escalas pode auxiliar no planejamento de inventários florestais e manejo, bem como minimizar impactos ambientais negativos (CONDÉ et al., 2016).

O desenvolvimento de softwares de Sistemas de Informações Geográficas (SIG) e funções de densidade probabilística como a função $\mathrm{K}$ e $\mathrm{F}$ de Ripley têm sido amplamente utilizados, pois explicam a variação da distância entre indivíduos (BRUZINGA et al., 2013; BATISTA et al., 2019; CAPRETZ et al., 2012; HIGUCHI et al., 2017). Entretanto, os estudos sobre a distribuição espacial de espécies geralmente levam em consideração apenas o tamanho da parcela e não a relação das distâncias entre os indivíduos, essas técnicas de análises espaciais associadas a pacotes estatísticos podem auxiliar na determinação da existência padrões espaciais relacionados a fatores ambientais e escala de diâmetro entre os indivíduos (ARAÚJO et al., 2014).

Este estudo permite gerar conhecimento para que as comunidades extrativistas possam desenvolver práticas de manejo mais sustentável para a B. excelsa, auxiliando em diversas aplicações, tais como: produção, qualidade e extração, transportação e armazenamento dos frutos.

Diante do exposto, o objetivo deste trabalho foi verificar qual o padrão de distribuição espacial da castanha-daamazônia (Bertholletia excelsa Bonpl.) em uma floresta nativa no sul do Amapá por meio da função de densidade probabilística K e F de Ripley.

\section{MATERIAL E MÉTODOS}

\section{Caracterização da área de estudo}

A Reserva Extrativista do Rio Cajari (RESEX Cajari) está localizada na região sul do estado do Amapá (Figura 1), instituída por decreto do governo federal em 1990 (BRASIL,
1990), com uma extensão de 481.650 ha que abrange três municípios, Mazagão, Laranjal do Jari e Vitória do Jari. No ano de 1997 o decreto federal de 30 de setembro redefiniu os limites da parte oeste da reserva, aumentando sua área para 501.711 ha.

Figura 1. Mapa de localização das parcelas na Resex do Rio Cajari, região Sul do estado do Amapá.

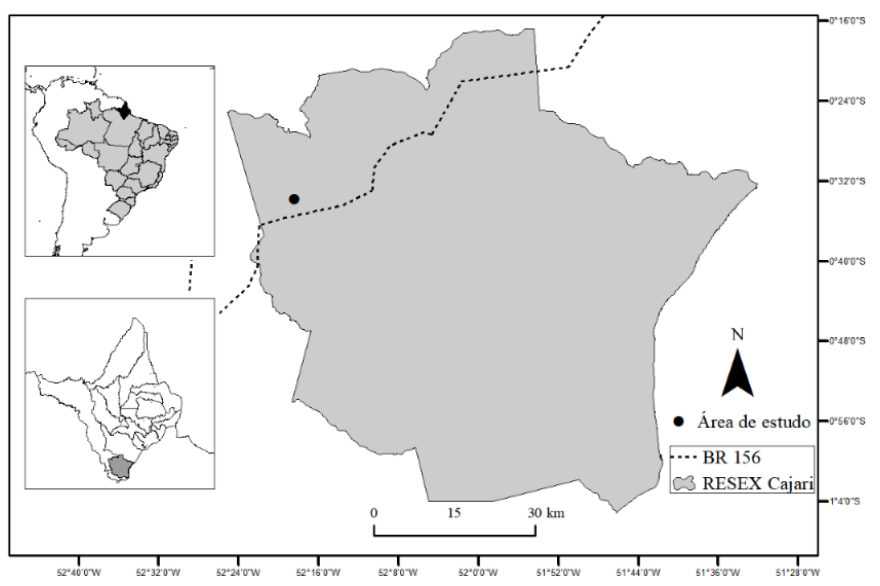

A específica área de estudo está inserida em uma região de alta densidade da espécie, denominados castanhais pelos extrativistas. Os locais amostrados podem ser considerados áreas de intensa coleta da semente (PAIVA et al., 2011). A vegetação na área de estudo, além das extensas áreas de castanhais, é composta por floresta ombrófila aberta, bem como áreas de transição cerrado-floresta.

O clima da região da RESEX Cajari situa-se na transição de clima tropical de savana (Aw) para tropical de monção (Am), segundo a classificação de Köppen. Apresenta temperatura média anual acima de $25^{\circ} \mathrm{C}$, com mínima de $18{ }^{\circ} \mathrm{C}$ e máxima de $31.5^{\circ} \mathrm{C}$. A precipitação anual em torno de $2.300 \mathrm{~mm}$, com concentração nos meses de dezembro a junho, e seca (precipitação < $100 \mathrm{~mm}$ por mês) de setembro a novembro. Na parte alta de terra firme da RESEX (Alto Cajari) onde foi realizado o estudo ocorrem os seguintes tipos de solo: Latossolos amarelo e vermelho amarelo, com ou sem ocorrência de concrecionário laterítico e uma mancha de Argissolo na face norte (RADAMBRASIL - Projeto Radar da Amazônia, 1974), com altitudes em torno de 150 metros.

Na região de extensos castanhais, foram implantadas duas parcelas permanentes de $300 \mathrm{~m}$ x 300 metros na Floresta Ombrófila Aberta e uma permanente na transição florestacerrado. Nos dois ambientes a distância mínima entre parcelas permanentes foi de $1 \mathrm{~km}$.

Foram medidos em cada ambiente todos os indivíduos de B. excelsa com diâmetro a 1,30 m do solo (DAP) $>10 \mathrm{~cm}$. As árvores mensuradas foram georreferenciadas, para confecção de um mapa de localização. Todos os indivíduos foram marcados com placas de alumínio, sendo enumerados de 
forma progressiva.

\section{Análise espacial pontual da castanha-da- amazônia}

Para verificar o padrão de distribuição espacial da espécie serão testadas as funções: F e K (RIPLEY, 1977).

\section{Função F}

A função $F$ é a função de distribuição acumulada da distância entre um ponto aleatoriamente escolhido (não o evento) e o evento mais próximo. Função das distâncias ponto-evento $\mathrm{F}(\mathrm{x})=\mathrm{P}(\mathrm{xi} \leq \mathrm{x})$.

O estimador simples para a função $F$ pode ser obtido pela equação:

$$
\widehat{F}(x)=\lambda^{-1} \sum_{i=1}^{m} I_{x}\left(x_{i}\right)
$$

Em que:

$\mathrm{m}$ : é o número de pontos arbitrários no mapa;

xi: representa a distância do i-ésimo ponto aleatório para o mais próximo dos $n$ eventos do mapa;

Ix (xi): uma função indicadora igual a 1 quando xi é menor ou igual a x e 0 caso contrário.

\section{Função $K$}

A análise da completa aleatoriedade espacial (CAE) é o primeiro passo na análise de padrões de pontos. Se a hipótese de aleatoriedade espacial for aceita, não há necessidade de se considerar um modelo mais complicado que e o modelo de Poisson. Também não é possível encontrar indicadores de interação interessantes entre os pontos (DIGGLE, 2003).

Foi aplicado o estimador de kernel smoothing (Gaussiana isotrópica) com largura de banda (bandwidth) que minimiza a soma de quadrados do erro obtidos por validação cruzada ("bw.diggle") para verificar se os fatores geradores da distribuição da densidade são homogêneos ou não, conforme recomendado por (TURNER; BADDELEY, 2005).

Para testar a hipótese de CAE foi utilizada a Função K de Ripley com correção do efeito de borda, como segue:

$$
\widehat{K}(h)=\frac{|A|}{n^{2}} \sum_{i=1}^{n} \sum_{i=1}^{n} \frac{I_{h}\left(u_{i j}\right)}{w_{i j}}
$$

Em que:

h: é o raio do círculo centrado em cada evento;

Ih(uij): é uma função indicatriz ou função peso, igual a 1 quando é menor que as distâncias h e 0 caso contrário;

n: é o número de eventos no mapa analisado com área $|A| ; e$

wij: é um fator de correção que representa a proporção da circunferência ao redor de um evento $\mathrm{i}$, passando sobre o evento j que está dentro de $|\mathrm{A}|$.

A função $\mathrm{K}$ teórica para um processo de Poisson homogêneo com área infinita é dada pela equação:

$$
K_{\text {pois }}(h)=\pi h^{2}
$$

Para facilitar a interpretação da função $K$ foi utilizado um estimador linear centrado em zero dessa função, denominada função $L$, conforme a equação abaixo:

$$
\widehat{L}(h)=\sqrt{\frac{\widehat{K}(h)}{\pi}}-h
$$

O raio máximo de busca (h), ou seja, a distância máxima foi dada pela regra de Ripley, que considera 0,25 da maior distância entre dois eventos na área de estudo, conforme Diggle (2003).

A CAE foi testada por meio de envelopes de confiança construídos por simulações de Monte Carlo para todas as funções testadas ( $\mathrm{F}$ e K). A análise foi feita de forma gráfica para facilitar a visualização dos desvios em relação à hipótese nula. Foram armazenados os valores máximo e mínimo da estimativa das funções para gerar os envelopes de confiança. O nível de significância é dado por $\alpha=1 /(s+1)$, em que s é o número de simulações.

As análises foram realizadas na plataforma (R Core Team., 2017), por meio do pacote Spatstat.

\section{RESULTADOS E DISCUSSÃO}

\section{Estatística descritiva}

Os valores encontrados para a média, moda e mediana mostraram que o comportamento da distribuição dos dados nas parcelas é de forma assimétrica positiva, ou seja, (média $>$ mediana > moda). A população de castanheiras apresentou elevados coeficientes de variação em todas as parcelas em estudo (Tabela 1).

No levantamento foram amostrados 55 indivíduos de $B$. excelsa no ambiente de transição cerrado-floresta (C), 29 e 85 
indivíduos nas duas parcelas em ambiente de floresta ombrófila aberta ( $\mathrm{A}$ e B), respectivamente conforme a (Figura 2), totalizando 169 árvores georreferenciadas para o estudo.

Figura 2. Distribuição dos indivíduos de Bertholletia excelsa Bonpl. na Resex do Rio Cajari, região Sul do Estado do Amapá. (A) e (B) ambiente de floresta e (C) transição cerrado-floresta.

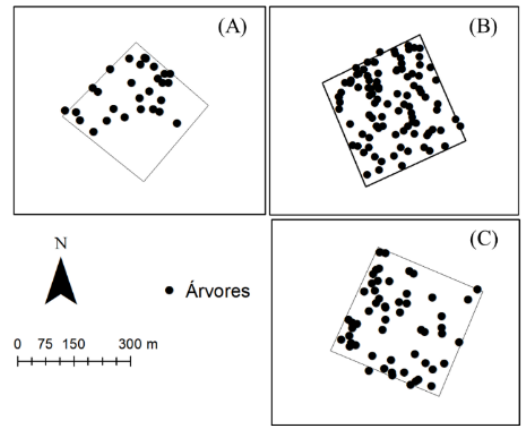

Foi observada predominância de indivíduos adultos (DAP $\geq 30 \mathrm{~cm}$ ) nas três parcelas, representando $86,2 \%$ na parcela 1 ; $91,8 \%$ na parcela 2 e $65,5 \%$ na parcela 3 com uma média de $81,17 \%$. Os indivíduos jovens (DAP < $30 \mathrm{~cm}$ ), em média, corresponderam a $18,83 \%$, sendo $13,79 \%$ na parcela $1 ; 8,2 \%$ na parcela 2 e $34,5 \%$ na parcela 3 .

\section{Análise das distribuições pelas funções $F$ e $K$ de Ripley}

A B. excelsa apresentou completa aleatoriedade espacial para todas as parcelas e em todas as distâncias testadas (60, 25 e $30 \mathrm{~m}$ ), respectivamente (Figura 3), onde os valores permaneceram entre os limites inferior e superior do envelope de confiança.

Os valores dispostos abaixo da linha do envelope indicam uniformidade, acima dessa linha, agregação e dentro dos limites do envelope, aleatoriedade (ARAÚJO et al., 2014).

A função L (linha cheia) e o envelope de confiança para 99 simulações (área em cinza) iniciam-se em zero, e assim como para a Função K a B. excelsa também apresentou completa aleatoriedade espacial para todas as parcelas estudadas (Figura 4).
Figura 3. Função $\mathrm{F}$ para indivíduos de Bertholletia excelsa Bonpl. na Resex do Rio Cajari, região Sul do estado do Amapá. (A) parcela 1; (B) parcela 2 e (C) parcela 3.
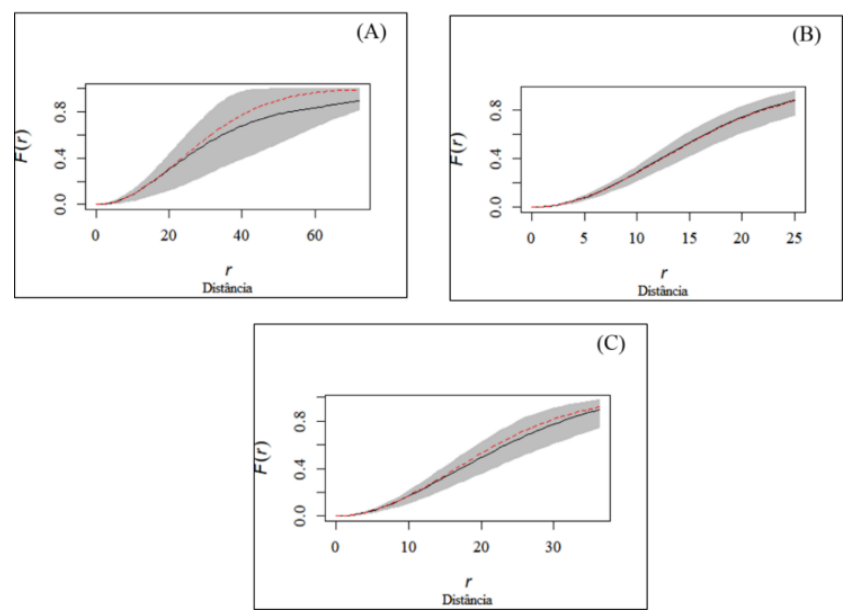

Figura 4. Função transformada $L(h)$ para indivíduos de Bertholletia excelsa Bonpl. na Resex do Rio Cajari, região Sul do estado do Amapá. Floresta ombrófila aberta (A) e (B), transição cerrado-floresta (C).

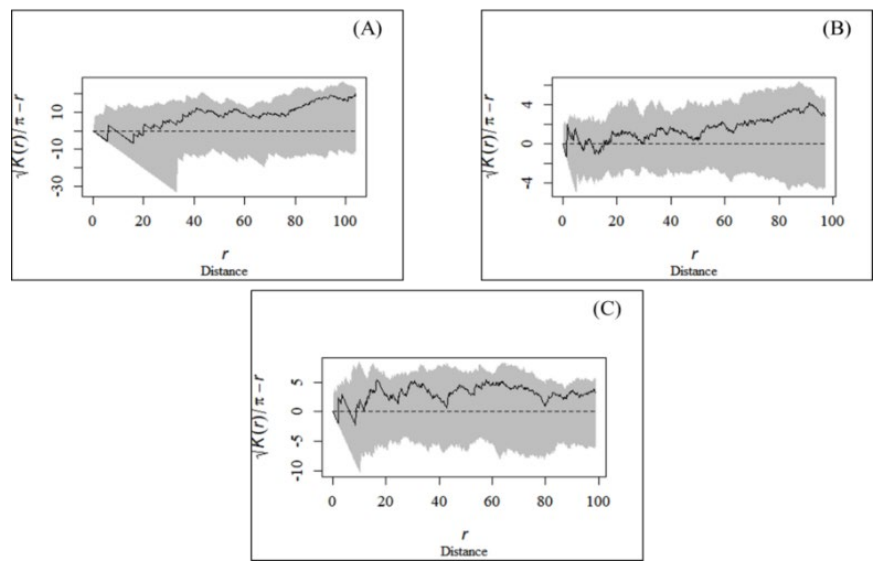

A elevada variação no diâmetro dos indivíduos de $B$. excelsa se deve a diferença entre o diâmetro de indivíduos jovens (DAP $<30 \mathrm{~cm}$ ) e adultos (DAP $\geq 30 \mathrm{~cm}$ ). O número de indivíduos jovens e adultos representam, em média, 18,83 e $81,17 \%$, respectivamente, do total de $B$. excelsa mensuradas nas parcelas.

Em um estudo realizado por Tonini (2008) também foi

Tabela 1. Estatísticas descritivas do diâmetro para a B. excelsa nas três parcelas na Floresta Ombrófila Aberta (A e B) e transição cerrado-floresta (C)na RESEX do Rio Cajari, Amapá. Em que: DAP é o diâmetro a altura do peito e CV\% é o coeficiente de variação em porcentagem.

\begin{tabular}{ccccccc}
\hline Parcela & $\mathbf{N}^{\mathbf{0}}$ de $\boldsymbol{B}$. excelsa & Média & DAP mín & DAP máx & Desvio Padrão & CV\% \\
\hline $\mathbf{1}$ & 29,0 & 73,9 & 20,1 & 170,3 & 35,1 & 47,5 \\
\hline $\mathbf{2}$ & 85,0 & 104,5 & 10,0 & 280,1 & 57,5 & 55,1 \\
\hline $\mathbf{3}$ & 55,0 & 78,5 & 10,0 & 253,4 & 62,8 & 79,9 \\
\hline TOTAL & 169,0 & - & - & - & - & - \\
\hline
\end{tabular}


observada grande predominância de indivíduos adultos (DAP $\geq 30 \mathrm{~cm}$ ), representando $64,7 \%$ na parcela 1 e 73,3\% na parcela 2, com uma média de $69 \%$. Os indivíduos jovens (DAP $<30 \mathrm{~cm}$ ), em média, corresponderam a 31\%, sendo 35,3\% na parcela 1 e $26,7 \%$ na parcela 2 .

\section{Fatores que influenciam na distribuição espacial da B. excelsa}

Plantas possuem necessidades diferentes conforme o seu estágio de vida e, em razão disso, apresentam padrões espaciais específicos. Condições ambientais como sombreamento, luminosidade, abertura no dossel da floresta, são algumas características fundamentais para o desenvolvimento de indivíduos jovens.

Indivíduos mais jovens tendem a ter distribuição espacial agregada (BRUZINGA et al., 2013), isso se deve ao fato de que indivíduos jovens estão na fase de recrutamento, enquanto que indivíduos adultos necessitam de uma maior cobertura para o seu crescimento e desenvolvimento de suas copas, e conforme ocorre o crescimento em diâmetro, ocorre o aumento de competição interespecífica por maior abertura do dossel florestal em busca de luminosidade (HART et al., 2018; JARDIM et al., 2007), com isso, poucos indivíduos alcançam grandes diâmetros, ficando mais distantes dos outros indivíduos e levando a uma distribuição espacial aleatória.

A distribuição espacial de uma espécie também pode ser determinada pela síndrome de dispersão das sementes, fenologia e polinização (CAPRETZ et al., 2012). A B. excelsa possui dispersão barocórica e zoocórica, devido ao tamanho dos seus frutos que podem pesar até $1,5 \mathrm{~kg}$ e o seu principal dispersor natural são roedores como a cutia (Dasyprocta sp.) (MARTINS et al., 2018) que se alimenta do ouriço e enterra as sementes, e esse processo é feito de forma aleatória. Com isso, mesmo as sementes sendo oriundas de árvores maiores de B. excelsa, a regeneração não irá acontecer próximo as árvores matrizes, o que levaria a formação de padrão agregado na ocorrência da espécie.

Dentre os trabalhos que buscaram compreender o padrão de distribuição espacial de indivíduos jovens e adultos, alguns encontraram padrão semelhante ao descrito neste trabalho, como (BRUZINGA et al., 2013) que estudando o padrão de distribuição espacial de indivíduos adultos de pequi encontraram padrão aleatório a partir da distância de 200 m entre os indivíduos. (HIGUCHI et al., 2017) encontraram padrão de distribuição aleatório para todas as espécies estudadas, com algumas tendendo a agregação. Outros detectaram padrões diferentes, como (CAPRETZ et al., 2012) que encontraram padrão agregado para as espécies em floresta ombrófila aberta e padrão aleatório para as espécies no cerrado a partir da distância de 120 m entre os indivíduos.

Nesse estudo não houve diferença na distribuição espacial da B. excelsa, o que pode justificar a semelhança na distribuição espacial da B. excelsa mesmo em ambientes diferentes como a floresta ombrófila aberta e a transição cerrado-floresta, é que os castanhais nativos na região da reserva extrativista do Rio Cajari são maduros e se encontram em estágio de grande produção por muitas décadas, levando em consideração que as castanheiras começam a produzir com DAP $>50 \mathrm{~cm}$. A grande quantidade de indivíduos adultos e a coleta intensiva de sementes por parte das comunidades extrativistas com o tempo veio diminuindo o desenvolvimento de novos indivíduos, o que contribuiu para a baixa ocorrência de indivíduos jovens nos castanhais.

O extrativismo não é uma prática que pode causar riscos a regeneração da $B$. excelsa, porque as comunidades realizam a coleta de sementes posteriormente a queda dos frutos, que ocorre no primeiro quadrimestre do ano, e esse tempo é necessário para que os dispersores naturais atuem, e como a distribuição das sementes é feita de forma aleatória é possível que muitas sejam depositadas em ambientes externos às parcelas de estudo. Uma vez que as cutias (Dasyprocta sp.) tem preferência em depositar as sementes em ambientes mais abertos, e este fato é denunciado pelo maior número de indivíduos jovens na parcela referente a transição cerradofloresta, que possui $34,5 \%$ de indivíduos com DAP $<30 \mathrm{~cm}$.

\section{CONSIDERAÇÕES FINAIS}

Em todas as parcelas do estudo predominaram as árvores adultas de $B$. excelsa.

As árvores de $B$. excelsa apresentaram distribuição espacial aleatória tanto em floresta ombrófila aberta quanto em transição cerrado-floresta.

A coleta intensiva de sementes por parte das comunidades extrativistas pode ter relação com a redução do número de indivíduos jovens nos castanhais, mas não afeta a dispersão natural da espécie.

\section{AGRADECIMENTOS}

Ao laboratório de Manejo Florestal, da Universidade do Estado do Amapá pelo suporte financeiro e a Empresa Brasileira de Pesquisa Agropecuária (EMBRAPA-AP) pela parceria no projeto

\section{REFERÊNCIAS}

ARAÚJO, E. J. G. DE; DAVID, H. C.; PÉLLICO, S.; MORAIS, V. A.; SCOLFORO, J. R. S. Padrão espacial de espécies arbóreas 
em fragmento de floresta estacional semidecidual. Revista de Ciências Agrarias - Amazon Journal of Agricultural and Environmental Sciences, v. 57, n. 2, p. 166-171, 2014.

BRUZINGA, J. S.; OLIVEIRA, M. L. R. DE; MACHADO, E. L. M.; LEITE, H. G.; PEREIRA, I. M.; NOGUEIRA, G. S. Distribuição espacial de indivíduos adultos de pequi. Scientia Forestalis/Forest Sciences, v. 41, n. 98, p. 249-256, 2013. BRASIL. Decreto N 99.145 de 12 de março de 1990, Criação da Reserva Extrativista do Rio Cajarí, 1990.

CAPRETZ, R. L.; BATISTA, J. L. F.; SOTOMAYOR, J. F. M.; CUNHA, C. R. DA; NICOLETTI, M. F.; RODRIGUES, R. R. Padrão espacial de quatro formações florestais do estado de são paulo, através da função $\mathrm{K}$ de Ripley. Ciencia Florestal, v. 22, n. 3, p. 551-565, 2012.

CONDÉ, T. M.; TONINI, H.; SILVA, F. DA; BARNI, P. E.; SOUZA CELES, C. H.; ARAUJO, R. F. DE; ASSIS CAMPOS, M. A.; CARNEIRO DE MIRANDA, D. L. Padrão espacial de espécies madeireiras da Amazônia pelo método de coordenadas cartesianas e espaciais. Pesquisa Florestal Brasileira, v. 36, n. 86, p. $115,2016$.

Diggle, P.J. 2003. Statistical analysis of spatial point patterns. Academic Press. 2da ed London, Arnold, 2003. 272p.

HART, S. P.; FRECKLETON, R. P.; LEVINE, J. M. How to quantify competitive ability. Journal of Ecology, v. 106, n. 5, p. 1902-1909, 2018.

HIGUCHI, P.; SILVA, A. C. DA; BERG, E. VAN DEN; PIFANO, D. S. Associações espaciais entre indivíduos de diferentes espécies de Miconia spp. Ruiz \& Pav. (Melastomataceae). Revista Árvore, v. 35, n. 3, p. 381-389, 2017.

JARDIM, F. C. DA S.; SERRÃO, D. R.; NEMER, T. C. Efeito de diferentes tamanhos de clareiras, sobre o crescimento e a mortalidade de espécies arbóreas, em Moju-PA. Acta Amazonica, v. 37, n. 1, p. 37-47, 2007.

LIMA, D.; MISAEL, C.; GODINHO, S. T.; BEZERRA, B. R.; VASCONCELLOS, R.; OLIVEIRA, G. L.; CUNHA, M. L.; ALVES, $X$. A. Estrutura e distribuição espacial de Symphonia globulifera $L$. $f$. em floresta de várzea baixa , Afuá-PA. Advances in Forestry Science, v. 5, n. 1, p. 275-281, 2018. MARTINS, K.; SANTOS, R. DA S. O. DOS; CAMPOS, T. DE; WADT, L. H. DE O. Pollen and seed dispersal of Brazil nut trees in the southwestern Brazilian Amazon. Acta Amazonica, v. 48, n. 3, p. 217-223, 2018.

NEVES, E. S.; GUEDES, M. C.; RODRIGUES, E. G. castanheiras, em capoeira e floresta da Resex Cajari Relationship of amazon nut ( Bertholletia excelsa Bonpl .) fruit production with the own variables, in fallow and forest Resex Cajari. n. 1990, p. 31-37, 2015.

NOGUEIRA, A. K. M.; SANTANA, A. C. DE. Influência das chuvas na oferta de castanha-do-brasil e o impacto no benefício socioeconômico e ambiental, no Oeste do estado do Pará. Desenvolvimento e Meio Ambiente, v. 45, p. 215-230,
2018.

PAIVA, P. M.; GUEDES, M. C.; FUNI, C. Brazil nut conservation through shifting cultivation. Forest Ecology and Management, v. 261, n. 3, p. 508-514, 2011.

BATISTA, A. P. B; SCOLFORO, H. F.; MELLO, J. M.; GUEDES, M. C.; TERRA, M. C. N. S.; SCALON, J. D.; GOMIDE, L. R.; SCOLFORO, P. G. V.; COOK, R. L. Forest Ecology and Management Spatial association of fruit yield of Bertholletia excelsa Bonpl . trees in eastern Amazon. Forest Ecology and Management, v. 441, n. March, p. 99105, 2019.

R CORE TEAM. R: A Language and Environment for Statistical Computing. ViennaR Foundation for Statistical Computing, 2017.

RADAMBRASIL - PROJETO RADAR DA AMAZÔNIA. Levantamento de recursos naturais. Rio de Janeiro: [s.n.]. TONINI, H.; EMILIO, P. com características morfométricas da copa e índices de competição. n. 1, p. 1509-1516, 2008.

TONINI, H.; IVANOV, G. B.; FLEIG, F. D. Fatores edafoclimáticos relacionados à produção de sementes em castanhais nativos de Roraima. Pesquisa Florestal Brasileira, v. 38, 28 dez. 2018.

TURNER, R.; BADDELEY, A. SPATSTAT: an $R$ package for analyzing spatial point patterns. Journal of Statistical Software, v. 12, n. 6, 2005.

\section{Submissão: 02/12/2019 \\ Aprovado para publicação: 04/03/2020}

